\title{
Optimal risk minimization of Australian energy and mining portfolios of stocks under multiple measures of risk
}

\author{
$\underline{\text { J. Arreola }}^{\text {a }}$ and R. Powell ${ }^{\text {a }}$ \\ ${ }^{\text {a }}$ School of Business, Edith Cowan University, Western Australia \\ Email: j.arreolahernandez.ecu.edu.au
}

\begin{abstract}
Australia's 2000's decade saw the sharpest rise in mining investments arising from developing Asian emerging economies' high demand for commodities like coal, iron ore, nickel, oil and gas which drove up prices to a historic level (Connolly \& Orsmond, 2011). As of December 2012, $39 \%$ and $9 \%$ of the Australian Securities Exchange's stocks were of the mining (coal and uranium stocks are included in this category) and energy (e.g. oil, gas and renewable energy stocks) sectors respectively, and investors recently have been considering separate portfolio positions in energy and mining stocks (Jennings, 2010). Facts of these nature set the stage for the task of selecting an optimal portfolio of stock securities where the fundamental questions faced by every investor, individual or institutional, are: a) what is the optimal point in time to go long in the investment position?, b) what are the optimal amounts to invest in every asset of a portfolio? and, c) when is the optimal time to short the portfolio investment position? The focus of the present study is on b) within a one period ahead forecast scenario.
\end{abstract}

Understanding the price and volatility movements of stock securities taking as a basis of study their own dynamics and co-dynamics is a complex task that may be better addressed through a multilateral modelling approach. This paper, in this regard, departs from a single model application by fitting multiple risk measures to the optimization of four portfolios each consisting of 20 ASX's stocks from the gold, iron ore-nickel, uranium-coal and oil-gas sectors. The five risk measures compared are: the variance, mean absolute deviation (MAD), minimizing regret (Minimax), conditional value at risk (CVaR), and conditional drawdown at risk $(\mathrm{CDaR})$, where the last two are threshold based measures. The risk measure parameters are input into meanvariance quadratic (QP) and differential evolution (DE) portfolio problem specifications.

Accurate estimations of the underlying interaction of stocks return series is a crucial element in portfolio allocation and portfolio risk management and frequentist traditional measures of dependence are rather inadequate. Here, with the objective of achieving more accuracy in the estimation of the dependence matrix, a Gaussian pair c-vine copula (PC), the regular graphical lasso (RL) and adaptive graphical lasso (AL) are fitted. Possible advantages from using these recently proposed and sophisticated techniques under model specifications where the covariance matrix is the measure of risk are indicated.

The main objectives of the present study are to calculate the optimal weights to be invested in every stock of the portfolios making use of linear and nonlinear model specifications and the risk measures suggested, analyse the weight allocation differences and seek portfolio optimization advantages from using pair vine copulas and the graphical lasso in the estimation of dependence. The present multimodal approach is, therefore, expected to be more robust and as a consequence, provide more complete information that could serve for improved decision making on portfolio selection, allocation and rebalancing. Research questions are answered based on the analysis of gold portfolio outcome values, only.

Findings indicate that $\mathrm{CDaR}$ is an important risk measure to be considered, along with other measures of risk when optimizing portfolios of stocks and no single measure of risk is suggested alone. The Gaussian pair cvine copula through the use of one different parameter in the modelling of every pair of variables' joint distribution appears to be more sensitive in capturing data's distribution characteristics. The adaptive graphical lasso also appears to be more perceptive when grasping the signal of the underlying interaction of the stocks. Therefore, valuable information could be drawn and inferred from applying multiple risk measures and sophisticated statistical techniques for their estimation. The weight allocation from threshold risk measures such as CVar and DaR and Minimax clearly differs from the rest. The models identified stocks with high return relative to risk and vice versa. The originality of the present study lies on the sectors of application and its multi-model nature.

Keywords: $\quad$ pair copulas, lasso, mean-variance quadratic, CVaR, CDaR, MAD, mining-energy stocks 
Arreola et al., Optimal risk minimization of Australian energy and mining portfolios of stocks under multiple measures of risk.

\section{INTRODUCTION}

Portfolio optimization has to do with optimal allocation of resources and the implicit management of risk which are modelled through a set of constraints and an element of forecasting. While constraints, as part of the model specification, shape the problem, the selected risk measure significantly influences the optimization outcome. The risk measures applied in this study could be considered as conservative investment approaches (perhaps suitable to risk averse investors preferring a lower return in exchange for minimal or no losses in their investments) whose priority is wealth preservation although not entirely.

Markowitz (1952) is recognized as the proposer of a nonlinear convex quadratic modelling technique for portfolio optimization. Under him, risk and return play a central role in the optimization problem where risk is identified as the variance to be minimized for a certain level of return. Risk under this setting is primarily linked to the covariance of the assets while diversification is highly sought. The inability of the method to account for extreme risk through the variance as the measure of risk and its tendency to generate implausible weight allocations (Allen, Kramadibrata, Powell \& Singh, 2012) has made the optimization of portfolios under a single risk measure largely obsolete while multi-risk measure-based portfolio optimization approaches, the norm. New statistical techniques for dependence matrix estimation with the purpose of improved portfolio optimization have been widely adopted and developed due to the possibility to exploit the multiple, already identified and parameterized, distribution functions of random variables according to their densities and corresponding probabilities, thus allowing, as in the case of pair copulas, the design of multivariate and multiform probability distribution structures based on those known distributions as the building blocks. The graphical lasso for sparsity generation and precision matrix estimation has grown in popularity primarily because it helps reduce the model's estimation error, often expressed as an error term in regression models, and the bias introduced by irrelevant-inactive variables (Tibshirani, Bien, Friedman, Hastie, Simon. Taylor \& Tibshirani J., 2012).

Portfolio optimization in a convex quadratic setting can be traced back to Markowitz (1952) with a large body of literature being devoted to extensions and applications of the model. A recent portfolio optimization adopting $\mathrm{CVaR}$ as the measure of risk with specific focus on the mining sector of Australia can be found in Allen and Powell (2010), which in line with Roy's Safety First criterion, minimizes the probability of attaining a return lower than the short fall probability. Recent endeavours in the field, on the other hand, have led to the development of a differential evolution algorithm (Ardia, Boudt, Carl, Mullen \& Peterson, 2011) able to solve convex and non-convex problems and account for nonlinearities in the distribution of the data. An implementation of the technique has been conducted by Krink and Paterlini (2011). Sklar's theorem, on the other hand, paved the road for what is now known as pair vine copula constructions for dependence matrix estimation, and Brechmann and Schepsmeier (2011) suggest Joe's (1997) work to be the first in dealing with pair vine copula constructions. Subsequent significant developments in the field have come through Bedford and Cooke (2001) and Aas, Czado, Frigessi and Bakken (2009). Finally, Dempster's (1972) work is considered, in the literature of sparsity search, to be a starting point towards Tibshirani's (1996) regular graphical lasso with subsequent improved versions of the model (e.g. adaptive graphical lasso) to be carried out by Fan and Li (2001) and Zou (2006).

The questions to be dealt with in this study are: What are the resulting weight allocation and weight allocation differences from applying multiple risk measures and models? And, do recent sophisticated methods for dependence matrix estimation such as pair vine copulas and graphical lasso generate any portfolio optimization advantages? Answer to these questions is based on the resulting gold portfolio values, only.

\section{MODELS}

The QP technique invokes the old saying of "not putting all one's eggs into one basket" and two underlying major assumptions of its framework are the normality of return's distribution and the quadratic utility expressing investors' preferences. The method is based on a central tendency (mean) measure that symmetrically and with escalating rate, due to the square feature of the quadratic objective function, penalizes deviations from the centre. The optimization problem to be solved here is:

$$
\min _{w} \frac{1}{n} \sum_{i=1}^{n}\left(\sum_{j=1}^{m} w_{j}\left(r_{i, j}-\mu_{j}\right)\right)^{2} \quad \text { s.t. } \quad \sum_{j=1}^{m} w_{j} \mu_{j}=\mu_{\mathrm{P}} ; \quad \sum_{j=1}^{m} w_{j}=1
$$

where $w_{j} \geq 0$, for $j=1, \ldots, m$ (Ghalanos, 2013). 
Arreola et al., Optimal risk minimization of Australian energy and mining portfolios of stocks under multiple measures of risk.

The MAD method, as compared to the QP, penalizes deviations from the centre with a linear rate. This feature, while allowing faster solutions to large optimization problems, does not adequately represent most investors' preferences and demands. However, since the risk measure does not scale or penalize highly kurtotic observations as heavily as the QP does, it could be considered as more robust. The linear problem to be solved here is:

$$
\begin{aligned}
\min _{w, d} \frac{1}{n} \sum_{i=1}^{n} d_{i} \quad \text { s.t. } & \sum_{j=1}^{m}\left(r_{i, j}-\mu_{j}\right) w_{j} \leq y_{i}, \forall_{i} \in\{1, \ldots, n\} ; \\
\text { s.t. } & \left.\sum_{\mathrm{j}=1}^{m} w_{j} \mu_{j}=\mu_{\mathrm{P}} ; \quad \sum_{\mathrm{j}=1}^{m} w_{j}=1 ; \quad r_{i, j}-\mu_{j}\right) w_{j} \geq-y_{i}, \forall_{i} \in\{1, \ldots, n\}
\end{aligned}
$$

with parameter $d_{i}$ accounting for absolute deviations from the forecast mean (Ghalanos, 2013).

The Minimax risk measure is perhaps the most conservative because, according to first constraint of the linear programming formulation (below), the difference between the maximum loss of the portfolio $M_{p}$ and the forecast return of the portfolio $\sum_{\mathrm{j}=1}^{m} w_{j} r_{i j}$ is targeted to be less or equal to zero (Ghalanos, 2013).

$$
\begin{array}{ll}
\min _{M_{p}, w} M_{p} \quad \text { s.t. } & M_{p}-\sum_{j=1}^{m} w_{j} r_{i j} \leq 0, \forall_{i} \in\{1, \ldots, n\} ; \quad \sum_{j=1}^{m} w_{j} \mu_{j}=\mu_{\mathrm{P}} ; \quad \sum_{j=1}^{m} w_{j}=1, \\
\text { s.t. } & w_{j} \geq 0, \forall_{j} \in\{1, \ldots, m\}
\end{array}
$$

$\mathrm{CVaR}$ has been suggested because it is a coherent measure of risk (e.g. when the probability distribution function is continuous) (Ghalanos, 2013) and is more in tune with the loss function of the tail distribution. The linear problem to be solved here is:

$\min _{w, d, v} \frac{1}{n a} \sum_{i=1}^{n} d_{i}+v \quad$ s.t. $\sum_{j=1}^{m} w_{j} r_{i, j}+v \geq-d_{i}, \forall_{i} \in\{1, \ldots, n\} ; \quad \sum_{j=1}^{m} w_{j} \mu_{j}=\mu_{\mathrm{P}} ; \quad \sum_{j=1}^{m} w_{j}=1$

s.t. $\quad w_{j} \geq 0, \forall_{j} \in\{1, \ldots, m\} ; \quad d_{i} \geq 0, \forall_{j} \in\{1, \ldots, n\}$

where $\mu_{\mathrm{P}}$ as above, represents the target return of the portfolio, $v$ is the VaR at the $a$-coverage rate, and $d_{i}$ accounts for the deviation values below VaR.

The CDaR measure of risk is path dependent and, in the context of portfolio optimization probable drawdowns instead of returns' distribution alone, are the focus of attention when estimating the optimal loadings on the stocks. The linear portfolio optimization problem to be solved here is:

$$
\begin{gathered}
\min _{w, u, v, z} v+\frac{1}{n a} \sum_{i=1}^{n} z_{i} \\
\text { s.t. } z_{i}-u_{i}+v \geq 0, \forall_{i} \in\{1, \ldots, n\} ; \sum_{j=1}^{m} w_{j} r_{i, j}+u_{i}-u_{i-1} \geq 0, u_{0}=0, \forall_{i} \in\{1, \ldots, n\} \\
\text { s.t. } z_{i} \geq 0, u_{i} \geq 0, \forall_{i} \in\{1, \ldots, n\} ; \sum_{\mathrm{j}=1}^{m} w_{j} \mu_{j}=\mu_{\mathrm{P}} ; \sum_{\mathrm{j}=1}^{m} w_{j}=1 ; w_{j} \geq 0, \forall_{j} \in\{1, \ldots, m\}
\end{gathered}
$$

with parameters $z$ and $u$ playing the role of auxiliary vectors of CDaR's variables and, for the purpose of modelling, the cumulative return distribution, respectively. The parameter $v$ accounts for the CDaR at the $a$ quantile level (Ghalanos, 2013).

The DE non-convex optimization technique uses floating-point encoding of a population, arithmetic operators and alteration for the selection and evolution of potential solutions and converges systematically by applying transformations (i.e. differential mutations) on vectors of parameters identified as the population. Analytically, let $x_{i, g}$ represent an existing population with $i$ vectors of parameters and $g$ generations. The first transformed or mutated vector of parameters $v_{i, g}$ is generated by randomly selecting three population members $x_{r 0, g}, x_{r 1, g}$ and $x_{r 2, g}$ or,

$$
v_{i, g}=x_{r 0, g}+F \cdot\left(x_{r 1, g}-x_{r 2, g}\right)
$$

where $F$ is a scaling factor with values greater than zero and less than one (Ardia et al., 2011).

On the subject of $\mathrm{PC}$, let the expression,

$$
P\left(X_{1} \leq F_{1}^{-1}\left(x_{1}\right), \ldots, X_{n} \leq F_{n}^{-1}\left(x_{n}\right)\right)=\mathrm{P}\left(U_{1} \leq x_{1}, \ldots, U_{n} \leq x_{n}\right) \equiv \mathrm{C}\left(x_{1}, \ldots x_{n}\right)
$$


Arreola et al., Optimal risk minimization of Australian energy and mining portfolios of stocks under multiple measures of risk.

where $F_{1}^{-1}\left(x_{1}\right)$ is the inverse distribution function, $X_{1}$ is a random variable, $U_{i} \equiv F_{i}\left(X_{i}\right)$ is uniformly distributed on $[0,1]$ and $C$ is a copula; if we make use of the property of inverse distributions $F_{i}\left(F_{i}^{-1}\left(U_{i}\right)\right) \geq$ $U_{i}$, divide the first term of the inequalities on both sides of (7) by $F_{i}$ and use Sklar's theorem (Sklar, 1959), the following equivalent expression of (7) should hold,

$$
F(\boldsymbol{x})=F\left(x_{1}, \ldots, x_{n}\right)=\mathrm{C}\left(F_{1}\left(x_{1}\right), \ldots F_{n}\left(x_{n}\right)\right)
$$

In the application of the Gaussian PC the model (9) suggested by Aas et al.(2009) for the decomposition of the multivariate probability density, is used.

$f\left(x_{1}, \ldots, x_{d}\right)=$

$\prod_{k=1}^{d} f_{k}\left(x_{k}\right) \cdot \prod_{i=1}^{d-1} \prod_{j=1}^{d-i} c_{i, i+j \mid 1:(i-1)}\left(\mathrm{F}\left(x_{i} \mid x_{1}, \ldots x_{i-1}\right), \mathrm{F}\left(x_{i+j} \mid x_{1}, \ldots, x_{i-1}\right) \mid \boldsymbol{\theta}_{i, i+j \mid 1:(i-1)}\right)$

Finally, estimation of the sparse dependence matrix through the RL and AL is accomplished by solving the following optimization problems:

$$
\begin{aligned}
& \log \operatorname{det} \Omega-\operatorname{tr}(\Omega \boldsymbol{S})-\lambda \sum_{i=1}^{n} \sum_{i=1}^{n}\left|w_{i j}\right| \\
& \log \operatorname{det} \Omega-\operatorname{tr}(\Omega \boldsymbol{S})-\lambda \sum_{i=1}^{n} \cdot \sum_{j=1}^{n} \varsigma_{i, j}\left|\omega_{i, j}\right|
\end{aligned}
$$

Here, $\boldsymbol{S}$ is the sample covariance matrix, $\Omega$ the precision matrix, $w_{i j}$ the matrix componenets of $\Omega, \lambda$ the penalizing or tuning parameter, det refers to the determinant, $t r$ refers to the trace, and $\varsigma_{i, j}$ in (11) is the penalty matrix of weights applied to the precision matrix components in the adaptive graphical lasso problem (Fan et al., 2009).

\section{DATA AND MODEL APPLICATION}

Samples (i.e. portfolios) of 20 daily frequency stock return series from the gold, iron ore-nickel, uraniumcoal and oil-gas from the ASX are modelled. The length of the return series spans from 7 January, 2005 to 2 July, 2012 thus covering the pre-GFC, GFC and post-GFC periods. The length of the return series may reduce the sampling error. All daily log return series have been filtered with an ARMA $(1,1)-G A R C H(1,1)$ with student-t innovations and, in the case of Gaussian PC copula application, a probability integral transform has been applied to the standardized residuals in order to obtain the copula data. The order of the columns in the PC application follows the criterion suggested by Czado, Schepsmeier and Min (2012), where the stock with the strongest relationship with the rest of the stocks in the portfolio becomes the first column in the data set and so on. The first column's places in the gold, iron ore-nickel, coal-uranium and oil-gas are occupied by St. Barbara (SBMX), BHP Billiton (BHPX), Paladin Energy (PDNX) and Woodside Petroleum (WPLX), respectively. The required $\mu_{P}$ has been set to be mean(colMeans(portfoliodata)) for mere convenience. All table's values are in percentage.
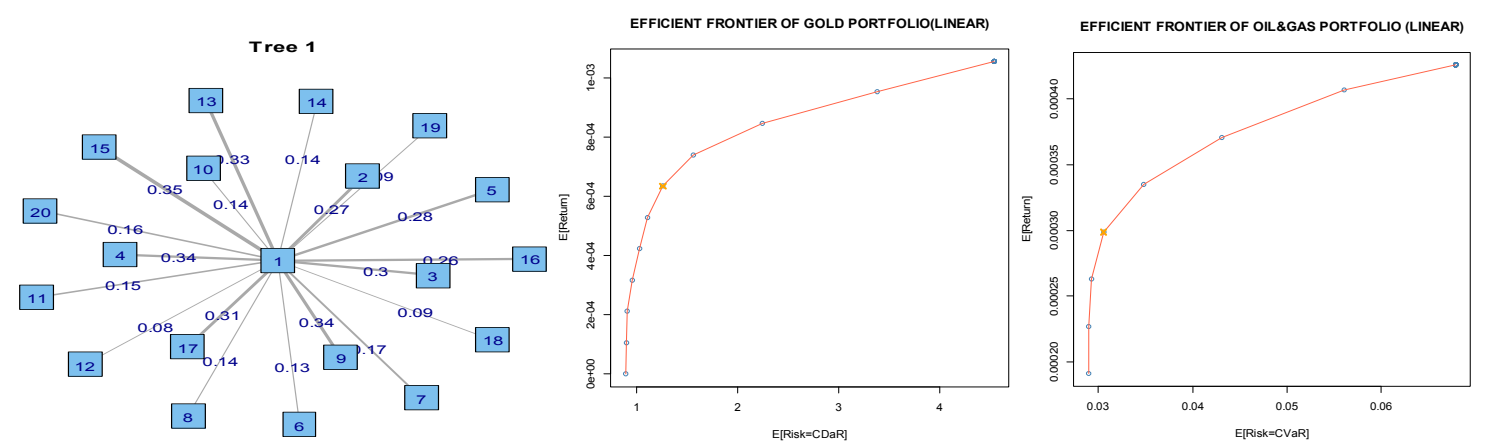

Figure 1: On the left, the first tree of applied Gaussian pair c-vine copula to the iron ore-nickel portfolio. On the centre and right, the efficient frontiers of gold and oil \& gas portfolios with CDaR and CVaR.

According to the gold portfolio's table of weights in the appendix, the CDaR measure of risk when optimizing does not seem to prioritize the overall historical distribution of returns per se, but instead the number of drawdowns occurred and those with the probability to take place in the future. The mean and variance are therefore under this framework necessary but not the most important factors. Concerning patterns in weight allocation, it can be seen that all risk measures on the model variations converge, with not very large variations from a mean of the weights, in Intermin Resources, Kalgoorlie Mining, Evolution Mining and Apex Minerals, indicating that these stocks have a high return relative to risk. It is also interesting to note that while under CDaR modelling St. Barbara (SBMX) (i.e. the stock most strongly correlated with the rest of the stocks in the gold portfolio) is given a weight of $13.22 \%$, the rest of the models 
Arreola et al., Optimal risk minimization of Australian energy and mining portfolios of stocks under multiple measures of risk.

do not follow, with CVaR, Minimax, QPPC, DEPC, DERL and DEAL placing near to zero weights while QPRL and QPAL give weights around 1.25\%. Another more or less consistent pattern of investment of the risk measures and models happens with Citigold where the only discrepant weight (e.g. zero) is allocated by CDaR.

Further analysis on the gold portfolio's table of weights indicates that, when the empirical covariance matrix of the log returns is used, the weights from the QP and DE are not very different. The weights under QPRL and QPAL and under DERL and DEAL are also quite alike. In QPPC and DEPC it can be seen that the Gaussian pair copula not surprisingly appears to capture characteristics in the data that QP, with the use of the empirical covariance matrix, does not. In fact, on multiple occasions when the QP allocates a high weight to a particular stock, the QPPC allocates a small amount. This type of opposed weighting behaviour makes sense under the well-known feature about QP of tending to underestimate the underlying interaction of variables. The QPAL and DEAL in comparison with the QP with empirical dependence matrix also shows an opposed weighting behaviour in many occasion indicating that the AL is more accurate in grasping the signal of the variables' network matrix. The main noticeable differences in weight allocation happen between the threshold risk measures (i.e. $\mathrm{CVaR}$ and $\mathrm{CDaR}$ ), Minimax and the rest of the models. The largest portfolio risk values are generated via $\mathrm{CVaR}, \mathrm{CDaR}$, Minimax, QPRL and QPAL, a possible indication that these model specifications are more perceptive to features in the tails of the data's distribution. In this application, the gold portfolio return is around $-0.10 \%$ where the negative sign is due to the targeted $\mu_{\mathrm{P}}$ being negative too. For a positive $\mu_{\mathrm{P}}$ it suffices to alter the targeted $\mu_{\mathrm{P}}$. Therefore, differences in the weight allocation based on the various risk measures and model specifications exist and the use of sophisticated techniques for dependence estimation such as pair vine copulas and the graphical lasso does generate different weight allocation that may lead to improved portfolio optimization. Finally, a risk contribution analysis based on the weight allocation under all risk measures and model variations indicates that De Grey Mining (DEGX), Intermin Resources (IRCX), Excalibur Mining (EXMX), Haoma Mining (HAOX) and Kalgoorlie Mining $(\mathrm{KMCX})$ are the largest contributors of risk to the gold portfolio. A table with risk contribution values has been omitted due to the space constraint.

\section{CONCLUSION}

Differences in weight allocation have been identified from all risk measures applied. Weight allocations derived from threshold risk measures such as $\mathrm{CVaR}$ and $\mathrm{CDaR}$ and, Minimax are noticeably different from those produced by the rest. $\mathrm{CDaR}$, based on its peculiar weight allocation criteria and the gold portfolio resource allocation in this study, is a risk measure to be considered and compared with alternative risk measures when optimizing portfolios of stocks. The use of a Gaussian pair vine copulas and adaptive graphical lasso models in the estimation of dependence generated weight allocations differing from the rest and when compared with that of QP, the models appear to capture more features in the tail's distribution. Specifically, the use of one different parameter in the modelling of every pair of variable's joint distribution by the Gaussian pair vine copula leads to difference results. The sparsity search technique of the adaptive graphical lasso, on the other hand, may have enabled a better grasp of the underlying signal of the dependence matrix. Therefore, multi risk measure approaches and the use of sophisticated techniques for the estimation of risk measures are suggested.

\section{REFERENCES}

Aas, K., Czado, C., Frigessi, A. and Bakken, H. (2009). Pair-copula constructions of multiple dependence. Insurance: Mathematics and Economics Journal, 44(2), 182-198.

Allen, D.E; Kramadibrata, A.R., Powell, R.J. and Singh A.K. (2012). Conditional value at risk applications to the global mining industry. Journal of Business and Policy Research, 7(3), 11-23.

Allen, D.E. and Powell, R.J. (2010). Measuring and optimizing extreme sectoral risk in Australia. School of Accounting, Finance and Economics. Edith Cowan University, 1-24.

Ardia, D., Boudt, K., Carl, P., Mullen, K. and Peterson, B. (2011). Differential evolution with DEoptim: an application to non-convex portfolio optimization. The R Journal, 3(1), 27-34.

Bedford, T. and Cooke, R.M. (2001). Probability density decomposition for conditionally dependent random variables modelled by vines. Annals of Mathematics and Artificial intelligence, 32, 245-268.

Brechmann, E.C. and Schepsmeier, U. (2011). Modelling dependence with c and d vine copulas: The Rpackage C-D vine. Journal of Statistical Software, 52(3), 1-26.

Connolly, E. and Orsmond, D. (2011). The mining industry: from bust to boom. Economic Analysis Department, Reserve Bank of Australia, 1-59. 
Arreola et al., Optimal risk minimization of Australian energy and mining portfolios of stocks under multiple measures of risk.

Czado, C., Schepsmeier, U. and Min, A. (2012). Maximum likelihood estimation of mixed c-vines with application to exchange rates. Statistical Modelling, 12(3), 229-255.

Dempster, A. (1972). Covariance selection. Biometrics, 157-175.

Fan, J. and Li, R. (2001). Variable selection via nonconcave penalized likelihood and its oracle properties. Journal of American Statistical Association. 96(456), 1348-1360.

Fan, J., Feng, Y. and Wu Y. (2009). Network exploration via the adaptive lasso and scad penalties. The Annals of Applied Statistics, 3(2), 521-541.

Ghalanos A. (2013). Portfolio optimization in parma (Version 1.5-0), 1-23.

Jennings, W.W. (2010). Energy stocks as a separate portfolio allocation. U.S. Air Force Academy, Colorado Springs, USA, 1-24.

Joe, H. (1997). Multivariate models and dependence concepts. Chapman and Hall, London.

Krink, T. and Paterlini, S. (2011). Multiobjective optimization using differential evolution for real- world portfolio optimization. Computational Management Science, 8,157-179.

Markowitz, H. M. (1952). Portfolio selection. Journal of Finance, 7, 77-91.

Sklar, A. (1959). Functions de repartition and dimensions et leurs marges. l'Institut de Statistique de L'Universit'e de Paris, 8, 229-231.

Tibshirani, R. (1996). Regression shrinkage and selection via the LASSO. Journal of the Royal Statistical Society, 58(1), 267-288.

Tibshirani, R., Bien, J., Friedman, J., Hastie, T., Simon., Taylor J., and Tibshirani R.J. (2012). Strong rules for discarding predictors in lasso-type problems. Journal of the Royal Statistical Society, 74(2), 245-266.

Zou, H. (2006). The adaptive lasso and its oracle properties. Journal of American Statistical Association, $10(1), 1418-1429$.

\section{APPENDIX A}

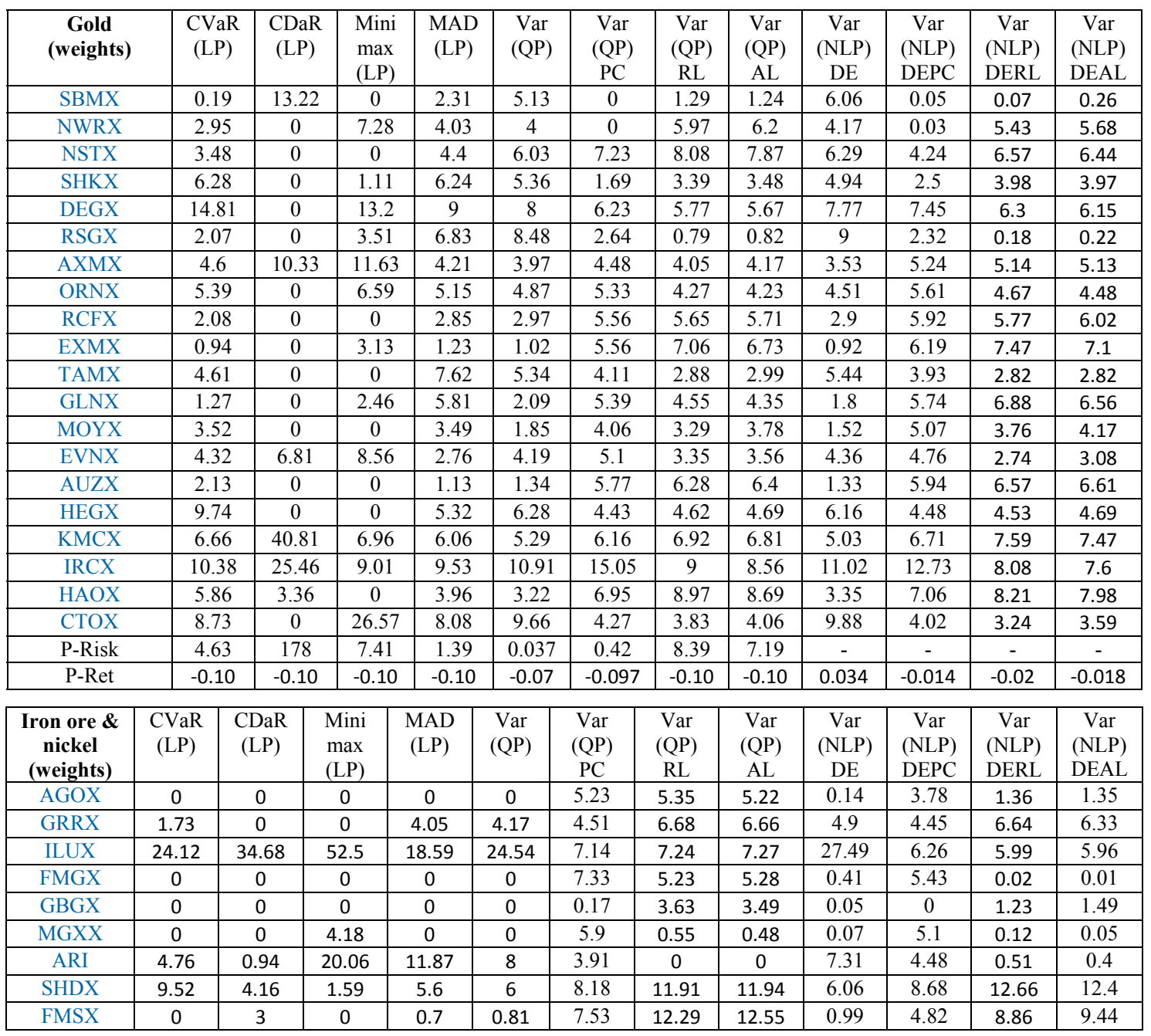


Arreola et al., Optimal risk minimization of Australian energy and mining portfolios of stocks under multiple measures of risk.

\begin{tabular}{|c|c|c|c|c|c|c|c|c|c|c|c|c|}
\hline MOLX & 3.34 & 0 & 0 & 0.97 & 0.66 & 4.86 & 2.72 & 2.63 & 0.23 & 6.17 & 6.8 & 7.14 \\
\hline ADYX & 0.86 & 0 & 0 & 1.89 & 1.4 & 6.08 & 6.82 & 6.77 & 1.56 & 7.78 & 9.92 & 10.02 \\
\hline ARHX & 0.46 & 10 & 11.82 & 1.96 & 1.02 & 5.19 & 3 & 2.87 & 0.71 & 7.03 & 7.57 & 7.57 \\
\hline BHPX & 31.6 & 38.41 & 9.64 & 32.36 & 37.1 & 0 & 0 & 0 & 30.76 & 0 & 0.02 & 0.01 \\
\hline FCNX & 4.12 & 8.69 & 0 & 3.34 & 2.5 & 4.16 & 4.7 & 4.99 & 2.31 & 7.33 & 10.18 & 10.07 \\
\hline IGOX & 0 & 0 & 0 & 1.43 & 0 & 6.69 & 3 & 3.22 & 1.05 & 5.73 & 1.49 & 1.52 \\
\hline POSX & 0 & 0 & 0 & 0.83 & 0 & 5.12 & 5.78 & 5.89 & 0.17 & 5.52 & 6.93 & 6.97 \\
\hline WSAX & 0 & 0 & 0 & 1.12 & 0 & 3.32 & 0 & 0 & 0.91 & 2.75 & 0.03 & 0.05 \\
\hline MLMX & 7.43 & 0 & 0.2 & 6.51 & 7 & 7.69 & 11.12 & 10.92 & 6.64 & 7.11 & 10 & 9.56 \\
\hline HRRX & 11.99 & 0 & 0 & 8.78 & 6.7 & 6.3 & 9.81 & 9.83 & 7.95 & 6.42 & 9.66 & 9.65 \\
\hline MCRX & 0 & 0 & 0 & 0 & 0 & 0.67 & 0 & 0 & 0.28 & 1.17 & 0 & 0 \\
\hline P-Risk & 4.18 & 53.40 & 8.26 & 1.26 & 0.03 & 0.68 & 18.31 & 16.65 & - & - & - & - \\
\hline P-Ret & -0.002 & -0.002 & -0.002 & -0.002 & 0.007 & -0.002 & -0.002 & -0.002 & 0.01 & -0.020 & -0.04 & -0.04 \\
\hline
\end{tabular}

\begin{tabular}{|c|c|c|c|c|c|c|c|c|c|c|c|c|}
\hline $\begin{array}{l}\text { Coal \& } \\
\text { Uranium } \\
\text { (weights) }\end{array}$ & $\begin{array}{c}\text { CVaR } \\
\text { (LP) }\end{array}$ & $\begin{array}{c}\mathrm{CDaR} \\
\text { (LP) }\end{array}$ & $\begin{array}{l}\text { Mini } \\
\max \\
(\mathrm{LP})\end{array}$ & $\begin{array}{l}\text { MAD } \\
\text { (LP) }\end{array}$ & $\begin{array}{c}\text { Var } \\
\text { (QP) }\end{array}$ & $\begin{array}{c}\text { Var } \\
\text { (QP) } \\
\text { PC }\end{array}$ & $\begin{array}{c}\text { Var } \\
(\mathrm{QP}) \\
\mathrm{RL}\end{array}$ & $\begin{array}{l}\text { Var } \\
(\mathrm{QP}) \\
\mathrm{AL}\end{array}$ & $\begin{array}{c}\text { Var } \\
\text { (NLP) } \\
\text { DE }\end{array}$ & $\begin{array}{c}\text { Var } \\
\text { (NLP) } \\
\text { DEPC }\end{array}$ & $\begin{array}{c}\text { Var } \\
\text { (NLP) } \\
\text { DERL }\end{array}$ & $\begin{array}{c}\text { Var } \\
\text { (NLP) } \\
\text { DEAL }\end{array}$ \\
\hline BWDX & 4.57 & 0 & 0 & 6.54 & 2.95 & 8.42 & 8.5 & 8.8 & 5.16 & 8.05 & 7.15 & 7.45 \\
\hline CNXX & 0 & 0 & 0 & 0 & 0.11 & 5.04 & 4.7 & 4.85 & 0.81 & 4.41 & 3.41 & 3.56 \\
\hline GLLX & 2.95 & 0 & 1.62 & 3.17 & 2.79 & 6.47 & 6.49 & 6.42 & 3.57 & 5.76 & 6.25 & 6.17 \\
\hline WALX & 0.97 & 16.94 & 0 & 1.91 & 1.92 & 7.19 & 10.15 & 10.06 & 2.22 & 7.08 & 8.55 & 8.43 \\
\hline BND & 2.41 & 0 & 0 & 1.48 & 1.34 & 5.29 & 4.23 & 4.37 & 1.33 & 5.09 & 4.37 & 4.44 \\
\hline CLAX & 3.78 & 0 & 0.97 & 11.22 & 0.48 & 1.41 & 2.8 & 2.78 & 0.52 & 2.45 & 7.32 & 7.37 \\
\hline NSLX & 1.83 & 0 & 0 & 1.88 & 0.9 & 5.84 & 5.12 & 5.09 & 1.54 & 6.31 & 6.53 & 6.47 \\
\hline AQAX & 0 & 21.14 & 0 & 0 & 0.98 & 2.23 & 3.69 & 3.17 & 3.17 & 2.44 & 1.05 & 0.59 \\
\hline NHCX & 3.97 & 30.39 & 0 & 6.49 & 13.36 & 5.11 & 5.34 & 5.22 & 16.77 & 4.43 & 3.28 & 3.42 \\
\hline SOLX & 51.87 & 0 & 55.21 & 49.34 & 58.77 & 5.16 & 6.3 & 6.58 & 46.03 & 4.69 & 4.72 & 4.8 \\
\hline BLZX & 2.67 & 0 & 6.58 & 1.46 & 1.18 & 7.29 & 6.42 & 6.38 & 1.14 & 8.65 & 8.23 & 8.23 \\
\hline FYIX & 8.6 & 0 & 14.48 & 5.09 & 3.7 & 6.24 & 6.51 & 6.51 & 3.19 & 7.17 & 8.76 & 8.76 \\
\hline SMMX & 0 & 0 & 4.5 & 0.19 & 4.94 & 8.36 & 7.68 & 7.71 & 4.22 & 6.69 & 3.99 & 4.08 \\
\hline EMAX & 5.63 & 0 & 0 & 3.22 & 3.15 & 4.13 & 0.34 & 0.34 & 3.05 & 3.98 & 1.07 & 1.12 \\
\hline PDNX & 0 & 0 & 0 & 0 & 0 & 0 & 0 & 0 & 0.48 & 0 & 0.02 & 0 \\
\hline UEQX & 0 & 0 & 7.23 & 1.52 & 0.84 & 4.68 & 3.94 & 3.9 & 1.4 & 4.77 & 4.14 & 3.98 \\
\hline LRRX & 2.22 & 0 & 2.34 & 0.3 & 0 & 1.57 & 4.17 & 4.44 & 1.24 & 1.79 & 6.26 & 6.34 \\
\hline DYLX & 0 & 0 & 0 & 0 & 0 & 5.08 & 4.29 & 4.45 & 1.36 & 4.27 & 2.2 & 2.31 \\
\hline BLRX & 0 & 0 & 0 & 0.05 & 0 & $\begin{array}{l}4.09 \\
\end{array}$ & 4.39 & 4.26 & 0.03 & 3.75 & 3.21 & 3.1 \\
\hline RSLX & 8.46 & 31.51 & 7.08 & 6.14 & 2.52 & 6.38 & 4.96 & 4.68 & 2.77 & 8.23 & 9.51 & 9.37 \\
\hline P-Risk & 3.37 & 103 & 6.26 & 0.945 & 0.015 & 0.5 & 10.01 & 9.34 & - & - & - & - \\
\hline P-Ret & $\begin{array}{l}-0.07 \\
\end{array}$ & $\begin{array}{l}-0.07 \\
\end{array}$ & $\begin{array}{l}-0.07 \\
\end{array}$ & $\begin{array}{l}-0.07 \\
\end{array}$ & -0.008 & -0.073 & -0.073 & -0.073 & 0.06 & -0.017 & -0.053 & -0.053 \\
\hline
\end{tabular}

\begin{tabular}{|c|c|c|c|c|c|c|c|c|c|c|c|c|}
\hline $\begin{array}{c}\text { Oil \& } \\
\text { Gas } \\
\text { (weights) }\end{array}$ & $\begin{array}{c}\mathrm{CVaR} \\
\text { (LP) }\end{array}$ & $\begin{array}{c}\mathrm{CDaR} \\
\text { (LP) }\end{array}$ & $\begin{array}{l}\text { Mini } \\
\max \\
\text { (LP) }\end{array}$ & $\begin{array}{l}\text { MAD } \\
\text { (LP) }\end{array}$ & $\begin{array}{c}\text { Var } \\
(\mathrm{QP})\end{array}$ & $\begin{array}{c}\text { Var } \\
(\mathrm{QP}) \\
\mathrm{PC}\end{array}$ & $\begin{array}{c}\text { Var } \\
(\mathrm{QP}) \\
\mathrm{RL}\end{array}$ & $\begin{array}{c}\text { Var } \\
(\mathrm{QP}) \\
\mathrm{AL}\end{array}$ & $\begin{array}{c}\text { Var } \\
\text { (NLP) } \\
\text { DE }\end{array}$ & $\begin{array}{c}\text { Var } \\
\text { (NLP) } \\
\text { DEPC }\end{array}$ & $\begin{array}{c}\text { Var } \\
\text { (NLP) } \\
\text { DERL }\end{array}$ & $\begin{array}{c}\text { Var } \\
\text { (NLP) } \\
\text { DEAL }\end{array}$ \\
\hline ACN & 7.68 & 0 & 0 & 5.89 & 1.64 & 4.19 & 5.84 & 5.5 & 1.21 & 5.23 & 7.72 & 7.32 \\
\hline AWEX & 6.76 & 6.27 & 0 & 2.76 & 0 & 0 & 0 & 0 & 0.27 & 0.03 & 0.01 & 0.22 \\
\hline BASX & 6.72 & 23.21 & 1.2 & 8.23 & 1.97 & 3.89 & 4.8 & 5.26 & 2.12 & 10.93 & 8.82 & 8.97 \\
\hline BPTX & 0 & 0 & 0 & 0 & 0 & 3.4 & 2.72 & 2.86 & 0.31 & 2.24 & 0.19 & 0.27 \\
\hline CTXX & 0 & 0 & 2.6 & 2.6 & 5.52 & 5.42 & 3.58 & 3.55 & 5.85 & 4.54 & 2.4 & 2.69 \\
\hline COEX & 0 & 0 & 0 & 0.75 & 4.73 & 7.04 & 8.2 & 8.23 & 5.1 & 5.3 & 5.61 & 5.63 \\
\hline CUEX & 5.49 & 3.59 & 18.64 & 5.92 & 4.22 & 5.45 & 6.33 & 6.48 & 4.6 & 5.28 & 6.37 & 6.31 \\
\hline GRVX & 3.29 & 0 & 0 & 5.71 & 1.94 & 9.56 & 15.33 & 14.71 & 1.78 & 10.7 & 16.19 & 15.73 \\
\hline LNGX & 0 & 0 & 0 & 0.24 & 0 & 5.3 & 4.83 & 5.1 & 0.04 & 4.59 & 3.7 & 3.86 \\
\hline MELX & 0 & 0 & 0 & 0.76 & 1.6 & 7.27 & 7 & 6.91 & 1.87 & 5.9 & 5.39 & 5.31 \\
\hline MOGX & 3.2 & 0 & 2.85 & 2.99 & 0.95 & 7.89 & 6.06 & 5.66 & 0.81 & 5.28 & 8.65 & 8.23 \\
\hline NXSX & 0 & 0 & 0 & 5.17 & 0 & 4.29 & 1.09 & 1.18 & 0.02 & 5.15 & 3.62 & 3.73 \\
\hline NWEX & 0 & 0 & 0 & 0.87 & 0 & 4.16 & 5.08 & 5.46 & 0.34 & 3.67 & 5.21 & 5.58 \\
\hline OEXX & 5.64 & 6.73 & 21.78 & 4.42 & 0.79 & 3.88 & 3.57 & 3.72 & 0.33 & 5.34 & 5.86 & 6.04 \\
\hline ROCX & 12.3 & 0 & 10.23 & 5.93 & 0 & 3.15 & 0 & 0 & 0.04 & 4.62 & 0.12 & 0.42 \\
\hline STOX & 0 & 0 & 0 & 3.96 & 1.87 & 4.07 & 0 & 0 & 1.66 & 3.67 & 0.03 & 0.08 \\
\hline TPTX & 1.91 & 0 & 1.41 & 0.88 & 2.02 & 9.01 & 14.41 & 14.27 & 2.13 & $\begin{array}{l}7.75 \\
7.75\end{array}$ & $\begin{array}{c}0.0 \mathrm{~J} \\
11.82\end{array}$ & 11.76 \\
\hline WPLX & 0 & 0 & $\begin{array}{c}1.41 \\
0\end{array}$ & 0 & 9.34 & 0 & 0 & $\begin{array}{c}14.21 \\
0\end{array}$ & 9.36 & 0 & 0 & 0 \\
\hline APAX & 24.9 & 14 & 0 & 25.34 & 30.9 & 6.42 & 7.64 & 7.53 & 30.78 & 5.28 & 6.19 & 5.95 \\
\hline ORGX & 22.04 & 46.13 & 41.28 & 17.56 & 32.46 & 5.6 & 3.52 & 3.57 & 31.38 & 4.5 & 2.11 & 1.89 \\
\hline P-Risk & 3.59 & 54.85 & 7.43 & 1.08 & 0.017 & 0.60 & 13.6 & 12.34 & - & - & - & - \\
\hline P-Ret & $\begin{array}{c}- \\
0.028\end{array}$ & -0.028 & -0.028 & -0.028 & 0.013 & -0.028 & -0.028 & -0.028 & 0.042 & -0.015 & -0.019 & -0.019 \\
\hline
\end{tabular}

